\title{
EINE VERGLEICHUNG VON SWEETS AUSGABE DES CODEX EPINALENSIS (FACSIMILE 1883, OET. 1885) MIT DER HANDSCHRIFT.
}

Einleitung.

Durch die güte des oberbibliothekars der Baseler universitäts-bibliothek, herrn dr. Bernoulli, nicht minder wie durch das freundliche entgegenkommen des herrn E. Douliot, bibliothekars der öffentlichen bibliothek zu Epinal, Frankreich, ${ }^{1}$ ) bin ich endlich in den stand gesetzt, meinen lange gehegten plan auszuführen, d. h. die handschrift selbst einzusehen und dieselbe mit Sweets veröffentlichung zu vergleichen. Das ergebnis dieser vergleichung lege ich hiermit vor. Vorausschicken will ich, dafs ich im spätsommer dieses jahres persönlich in Epinal war, um auszufinden, warum ein früherer versuch fehlgeschlagen war, den ich von Leiden aus gemacht hatte, die hs. in meine hände zu bekommen. Es stellte sich heraus, dafs dies an der fehlerhaften angabe der nummer der hs. lag, die ich auf grund von Sweets angabe ${ }^{2}$ ) (Facsimile Introd. p. V a) als nr. 17 bezeichnet hatte. Die hs. hat diese nummer nie getragen. Auf der rückseite des vorlegeblattes findet sich in schwarz von derselben hand, die die angabe über ursprung der hs. auf der ersten seite macht, oben der vermerk: $A R M^{r}{ }^{3}$ ) $\mathrm{nr} .7$; darunter in roter tinte: no. 66. Auf dem ersten pergamentblatte ist oben linker hand ein kleiner viereckiger streifen

1) Herr dr. Bernoulli war sofort bereit, meinem antrage stattzugeben und die hs. auf diplomatischem wege mir zu verschaffen. Herr Douliot hat in der liebenswürdigsten weise das seine dazu beigetragen, dals die zeitweilige versendung dieses schatzes der Epinaler stadtbibliothek von dem bürgermeister der stadt und der hohen französischen regierung gewährt wurde und die hs. ohne allzu lange verzögerung hier eintraf. Dafür sei ihnen allen der gebührende dank abgestattet.

2) Er hat damit nur eben kopiert, was Mone, der erste herausgeber des Epinal, im Anzeiger f. d. Kunde d. teutsch. Vorzeit, Jahrg. 7, 133, irrtümlich berichtet. Auch seine angabe der herkunft der hs. entstammt augenscheinlich dem, was Mone a. a. o. darüber sagt.

3) d. h. Armoir 'schrank'. 
anfgeklebt, ein gleicher oben anf dem rücken des ledereinbandes; beide tragen die gedruckte, also moderne $\mathrm{nr}$. 72, und das ist die nummer, unter der die hs. heute im kataloge eingetragen ist. Die ursprüngliche nummer war nach den erklärungen des herrn E. Douliot nr. 7.1) Bei einer neuordnung erhielt unser ms. die nr. 66. Eine weitere umordnung in moderner zeit führte zu der bezeichnung durch $\mathrm{nr}$. 72. Der bibliothekar war bei meinem ersten besuche nicht wenig erstaunt, als ich ihm Sweets facsimile zeigte. Von dem vorhandensein eines solchen hatte er nicht die geringste ahnung. Er erklärte, kein exemplar desselben sei in der öffentlichen bibliothek vorhanden. Es scheint also, dals Sweet unterlassen hat, seinen dank an die Epinaler bibliothek durch überreichung eines exemplars seines facsimiles abzustatten. Auch die OET. waren nicht da.

Der Codex Epinalensis nr. 72 entstammt, wie Mone-Sweet angegeben haben, dem kloster Moyen-Moutier, trug da augenscheinlich 1) die nr. 19 anno 1717. Das scheint wenigstens aus dem vermerke einer hand des 18. jahrh. anf der ersten seite der hs. hervorzugehen, der besagt: Mediani Monafterij Catalogo infcriptus. 1717. Darunter steht von derselben hand: Sermones xLvirr. S. Auguftini Epifcopi. Diese angabe ist irrtümlich, denn der Sermones sind 49, wie der schreiber der hs. selbst in der folgenden zeile angibt: INCIPIVNT CAPI-

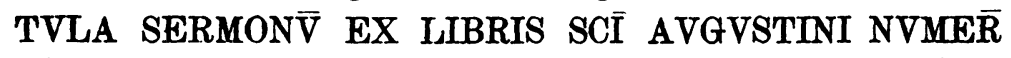
xLvirj. Der schreiber des Codex der Sermones gibt seinen namen auf der ersten zeile an als Johannes und bezeichnet als seinen mitarbeiter Rehgar. Der Codex ist nach ihm de majori ecclesia :

Ille tıb' è de majozı ecl'za ezo joh'el fecr cū rehzarj Auf der vorder- und rückseite des ersten blattes und den ersten vier zeilen des nächsten blattes steht die inhaltsangabe. Der text beginnt auf blatt 2, geschrieben von zwei händen, und läuft durch bis zum ende von blatt 93 . Die blätter waren unnumeriert; nur hier und da hatte Mone eine nummer bei-

1) Demnach wäre also die ausgestrichene nr. 110 nicht von dem belang, den ich anfangs anzunehmen geneigt war. Ich vermutete, die ansgestrichene nr. 110 war die ursprüngliche and warde ausgestrichen und 7 dafür gesetzt, als eine neuordnung vorgenommen wurde. Die Moyen-Montier-nummer findet sich auf der innenseite des vorderen bachdeckels. Sie ist, in starker schrift mit resten von goldsand: x. 1. darunter nr. 19. 
geschrieben; ich habe die paginiernng vervollständigt. An diesen Augustinustext schliefst sich dann auf 14 folioblättern das glossar an, das, wie schon die schrift zeigt, bedeutend älter als der vorhergehende text ist. Dieser text ist nach einem vermerk auf der rückseite des vorlegeblattes im 11. jahrhundert geschrieben. Welchem jahrhundert das glossar angehört, mufs noch ermittelt werden. Nach Sweets facsimile-ausgabe, einleitung, p. XI b, 'Mr. Thompson, head of the Mss. department of the British Museum, unhesitatingly put down Epinal as early ninth century, and still maintains that opinion after a further examination, speaking, of course, only as a palæographist, and without taking the linguistic evidence into consideration.' Sweet kommt p. XIV zu dem schlusse 'that Epinal was probably written not later than the beginning of the eight century, being a copy of an older Ms. of undeterminable date'. Ob diese frühe datierung haltbar ist, werden wir später des nähern sehen. Ganz unhaltbar scheint mir, das will ich gleich hier bemerken, das datum, unter dem das NED. für gewöhnlich den Epinal ansetzt, nämlich vor 700. Erst neuerdings hat sich Craigie in der July 1, 1908 nummer dazu verstanden, das datum zwei jahrhunderte herunterzurücken und damit der wahrscheinlichkeit näher zu kommen, indem er unter rib sb. ${ }^{2}$ das datum als circa 825 angibt ${ }^{1}$ ) und damit also zu dem ansatze Mones zurückkehrt, der im Anzeiger f. d. Kunde d. deutsch. Vorzeit jhrg. 7, $133^{5}$ das Epinaler glossar der ersten hälfte des 9. jahrh. zuweist. Die frage der altersbestimmung des glossars wird, wie gesagt, später ausführlich erörtert werden. Wir können uns einstweilen begnügen festzustellen, dals Sweet recht hat, wenn er p. V a der einleitung sagt, dals 'the connection of the glossary with the text which precedes it is purely external'. Ob aber die vereinigung der beiden ungleichaltrigen

1) Murray dagegen im Oktober 1910 kehrt unter table sb. 11 zu der fruthen datierung 'vor 700' zurück. Sie scheint schon wegen der von mir nachgewiesenen zahlreichen glossierung aus Aldhelm unmöglich. Die nachweise dafur sind im Corp. Gloss. Latin. VII zu finden und ron W. Heraens in seiner schrift 'Die Sprache des Petronius' anerkannt. Auch gegen die datierung vor 700 sprechen die Vita St. Guthlaci-glossen, von denen eine ganz sichere ich hier anführen will: spatula (d. h. spatalo) [aus psiathulo] bed geht auf Vite St. Guthlaci ed. Gonser (Angl. Forsch. heft 27) p. 110 zurtick, wo es heifst: ... tunc indutos artus agresti de spatulo surgens arrexit. Die Vita entstand im 8. jahrh., nach Gonser zwischen 716 und 749. 
bestandteile erst in moderner zeit durch den buchbinder bewerkstelligt worden ist, wie Sweet des weiteren meint, möchte ich denn doch bezweifeln. Der band sieht ziemlich alt aus. Vielleicht stammt er noch aus der zeit, da die ehemalige abtei Moyen-Moutier den Codex eignete. Auf dem rücken steht in golddruck oben: D. AVGVSTI | SERMONES | XLVIII Unten ist aufgeschrieben in tiefschwarzer schrift das katalogdatum 1717, d. h. das jahr, wo die hs. im genannten kloster inventarisiert wurde laut vermerk auf bl. 1; die ersten drei ziffern sind noch deutlich zu erkennen, die vierte ist verkleistert. Man beachte, daIs die zahl der Sermones fälschlich als 48 statt 49 angegeben ist. Dals die 93 pergamentblätter der Sermones und die 14 des glossars ursprünglich heterogen sind, geht auch aus ihrer ungleichen länge und breite hervor. Seine grölsere breite ${ }^{1}$ ) hat der buchbinder dem glossar gelassen. Gleichförmigkeit der länge hat er aber durch beschneiden oben herbeigeführt und dabei leider einen vermerk verstümmelt, der über der ersten zeile des glossars stand und sich in der breite der sechs kolumnen erstreckte. Was der vermerk besagen wollte, lälst sich aus dem stehen gebliebenen zwar schwer erkennen. Doch ist es mit dr. Bernoulli's hilfe gelungen, ihn als folgendermalsen lautend $\mathrm{zu}$ lesen: Lexicon Anglo-saxonicum vetus. vide, bene ${ }^{2}$ ) suade ${ }^{2}$ ), Hickesij thesaurum. Da der Thesaurus 1703 erschien, so wird der vermerk in die zeit zwischen 1703 und 1717 zu setzen sein. Sweet sagt auch nicht ein wort darüber und doch war eine bemerkung sehr angebracht. Der ahnungslose leser könnte ohne dieselbe z. b. glauben, rechts von fantasiā (so hs.) zeile 1, kolumne B, ständen noch zwei buchstaben, $c^{v}$; es ist aber nur unterer rest des abgeschnittenen $\mathrm{g}$ von Anglo-.

Notiz: Ich benutze die gelegenheit, darauf hinzuweisen, dafs Ms. 40 der Trierer stadtbibliothek (siehe ZfdA. 52, 169 ff.) auch für den Epinal wichtig ist. Zu a.a.0., s. $169^{29}$, bemerke ich, dafs fol. 55 r. Alea. tafle, Aleator. tafleri, Albugo. fli, fol. 56 r. Alba spina. haguthorn etc. steht. Mehr später.

1) Die blätter ragen fast einen halben zoll über die der Sermones hervor.

2) be? diese beiden worte konjektur, nur eo sicher.

Basei, November $1910 . \quad$ Otto B. Schlutter. 